\title{
Efeito da adição de resíduo do endocarpo de tucumã (Astrocaryum Aculeatum) em Poliestireno de Alto Impacto (PSAI)
}

\author{
Effect of adding endocarp residue tucumã \\ (Astrocaryum Aculeatum) in High Impact \\ Polystyrene (HIPS)
}

Andrey Marcos Pinho da Silva' ${ }^{1}$, Daniel Esquerdo Margalho ${ }^{2}$ Darbens Silvio Correia Junior ${ }^{2}$

\footnotetext{
${ }^{1}$ Departamento de Engenharia de Materiais - Faculdade de Tecnologia - UFAM, CEP: 69067-005, Manaus, AM, Brasil. ${ }^{2}$ Programa de Pós-Graduação em Ciência e Engenharia de Materiais - PPGCEM/UFAM, CEP: 69067-005, Manaus, AM, Brasil

e-mail: andreymarcos@ufam.edu.br, danielmargalho@gmail.com
}

\section{RESUMO}

Este trabalho investigou o efeito da adição de resíduo do endocarpo de tucumã (Astrocaryum Aculeatum) em poliestireno de alto impacto. Foram obtidos corpos de prova por meio do processamento de injeção plástica, para a confecção dos corpos de prova, foram utilizados $2 \%$ e $4 \%$ em peso de resíduo (CPS2 e CPS4). Foram realizadas caracterizações térmicas (TG e DSC), mecânicas (ensaio de impacto e resistência em módulo de tração) e um estudo com espectrometria de infravermelho (FTIR). Portanto o estudo mecânico indicou uma menor resistência ao impacto e tração dos CPS2 e CPS4 comparados com o material puro (PSAI). O estudo térmico indicou que a estabilidade térmica é aprimorada com a incorporação do resíduo. Por meio da espectrometria de infravermelho (FTIR), foi possível verificar a perda de ligações de monossubstituição. De forma geral, foi possível observar uma boa adesão entre as partículas, notou-se o efeito compatibilizante da lignina residual, as interações resíduo-polímero provocaram alterações nas propriedades térmicas, mecânicas e espectroscópicas nos corpos de prova.

Palavras-chave: tucumã, termoplásticos, resíduo, comportamento mecânico, análise térmica.

\begin{abstract}
This work investigated the effect of adding residue from the tucumã (Astrocaryum Aculeatum) endocarp in high impact polystyrene. Specimens were obtained through plastic injection processing, to make the specimens, $2 \%$ and $4 \%$ by weight of waste (CPS2 and CPS4) were used. Thermal (TG and DSC), mechanical (impact test and tensile strength tests) and a study with infrared spectrometry (FTIR) were performed. Therefore, the mechanical study indicated a lower impact and traction resistance of CPS2 and CPS4 compared to pure material (HIPS). The thermal study indicated that thermal stability is improved with the incorporation of the residue. Through infrared spectrometry (FTIR), it was possible to verify the loss of monosubstitution bonds. In general, it was possible to observe a good adhesion between the particles, the compatibilizing effect of the residual lignin was noted, the residue-polymer interactions caused changes in the thermal, mechanical and spectroscopic properties in the specimens.
\end{abstract}

Keywords: tucumã, thermoplastics, residue, mechanical behavior, thermal analysis. 


\section{INTRODUÇÃO}

O elevado consumo de materiais plásticos se tornou uma grande problemática, há um grande interesse por novas tecnologias que possibilitem a reciclagem e a produção de materiais com um menor impacto ambiental. Neste sentido os compósitos plásticos de madeira (WPC) receberam considerável atenção nos últimos anos. Esta tecnologia possibilita a reutilização de materiais orgânico, derivados de madeira, incorporando em outros materiais da classe de polímeros ou cerâmicas. Os WPCs vêm sendo aplicados em vários ramos de comércio, incluindo revestimentos, telhados, janelas, caixilhos de portas e indústria de móveis para ambientes externos [1-3].

Neste contexto, a região amazônica é repleta de possibilidades ainda não exploradas pelo homem. Do ponto de vista científico, são inúmeras espécies de animais e plantas que ainda não possuem estudos específicos que possam extrair seu verdadeiro potencial. Deste os diversos materiais alguns podem chamar a atenção para aplicações tecnológicas, como é o caso das fibras de curuá e açaí na fabricação de painéis [4], ou até mesmo no estudo de estruturas da escama do pirarucu (Arapaima gigas), que possui um mecanismo complexo de flexibilidade e elevada resistência à tensão [5].

Portanto a Amazônia com sua biodiversidade tem muito em contribuir para um avanço tecnológico [6, 7]. Dentre uma vasta potencialidade um material bastante comum na cultura popular amazônica chama a atenção é o tucumã (Astrocaryum Aculeatum) (Figura 1), é uma palmeira, que pertence à família das Arecacea, pode alcançar até 15 metros de altura, produz um fruto elipsoide e amarelado sua parte comestível (polpa) varia de 2 a $5 \mathrm{~cm}$, sua parte interna é composta de uma amêndoa e seu endocarpo possui características lenhosas $[8,9]$. O tucumã é um fruto lignocelulósico, contém em seu endocarpo estruturas como a hemicelulose, celulose e lignina. $[9,10]$.

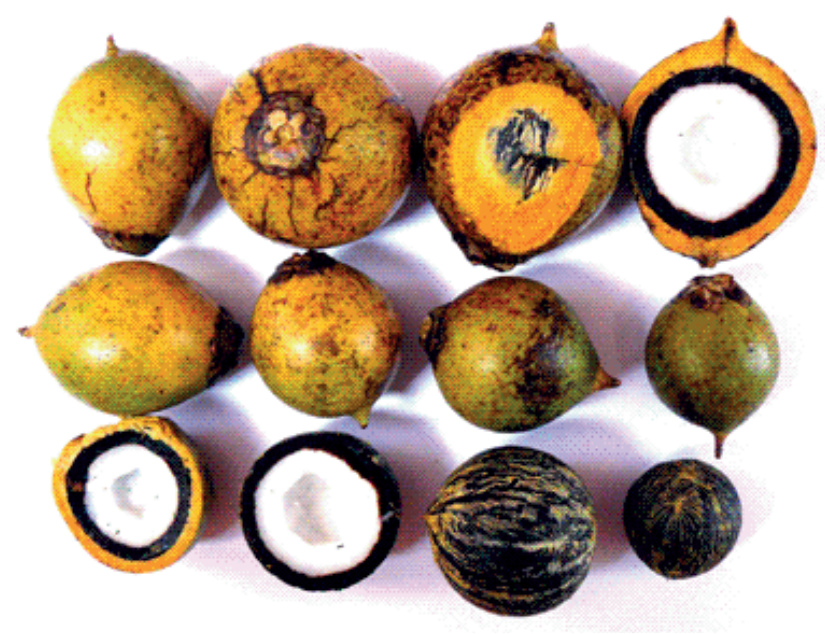

Figura 1: Fruto de tucumã (Astrocaryum Aculeatum).

Dentro dos polímeros estirênicos, existe o poliestireno de alto impacto (PSAI), um termoplástico que possui boa flexibilidade e boa maleabilidade, sendo geralmente utilizado em aplicações que não exijam longa durabilidade. É um material leve, flexível e opaco, com variadas aplicações. O poliestireno de alto impacto (PSAI) possui em sua composição, cerca de $14 \%$ de polibutadieno o que garante uma significativa tenacidade, ductilidade e resistência ao impacto comparado ao poliestireno cristal $[11,12]$. Tem aplicação em displays de produtos em geral, placas de sinalização internas, brindes e embalagens de produtos $[12,13]$.

A combinação de materiais vegetais com termoplásticos é algo visto como grande potencialidade, no que tange critérios de sustentabilidade de novos materiais, algumas fibras já tiveram sua potencialidade evidenciada, como as fibras de coco, sisal, bagaço de cana-de-açúcar, cânhamo e algodão [14, 15]. O presente artigo visa difundir o uso de resíduos vegetais como aditivos poliméricos em termoplástico. Portanto foi investigado o efeito da adição de resíduo do endocarpo do tucumã (Astrocaryum Aculeatum) com o poliestireno de alto impacto (PSAI), utilizando a injeção plástica como forma de processamento dos materiais e analisando sua eficácia por meio de análise espectroscópica (FTIR), térmica (TG e DSC), mecânica (resistência ao impacto e tração). 


\section{MATERIAIS E MÉTODOS}

\subsection{Coleta e tratamento do tucumã (Astrocaulym tucuma)}

Os resíduos de tucumã (Astrocaulym tucuma), fruto nativo da floresta amazônica (figura 2), foram obtidos como matéria-prima a partir do seu ouriço (endocarpo). Esses ouriços foram coletados em feiras comerciais da cidade de Manaus, Amazonas no mês de novembro de 2017. Após a coleta o material foi submetido a um processo de secagem por 48 horas em $60{ }^{\circ} \mathrm{C}$. Posteriormente ocorreu a cisão do endocarpo a fim de separar o endocarpo e a amêndoa.

Subsequentemente o endocarpo foi triturado em um moinho de facas, durante 30 minutos. O produto do moinho foi peneirado, utilizando apenas a fração passante em 40 mesh.

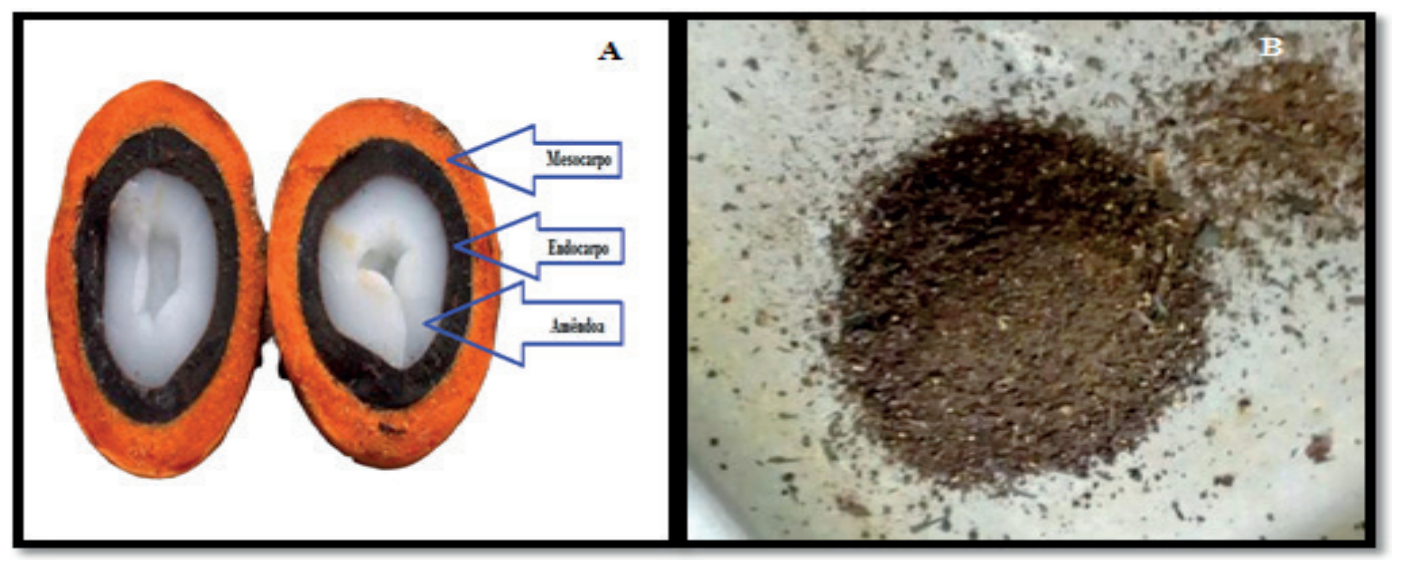

Figura 2: Representação do fruto (Astrocaryum aculeatum). Figura 1.A demonstra o esquema da composição da estrutura do tucumã (Astrocaryum aculeatum). Figura 1.B demonstra o endocarpo do tucumã (Astrocaryum aculeatum) triturado.

\subsection{Obtenção do poliestireno}

O poliestireno de alto impacto foi cedido pela empresa Videolar-Innova S/A. Unidade IV, situada na Av. Abiurana, 1616 Distrito Industrial, Manaus-AM.

\subsection{Preparação dos materiais}

Foram preparadas duas proporções de resíduo/polímero A primeira foi a proporção de $98 \%$ de PSAI e 2\% de resíduo de tucumã em peso e a segunda foi uma proporção de $96 \%$ de PSAI e $4 \%$ de resíduo de tucumã.

\subsection{Obtenção dos corpos de prova}

Os corpo de prova e tração e impacto izod foram obtidos na injetora (marca KraussMaffei, tipo 65-135 C1 de 1995) da empresa Videolar-Innova S/A. Os corpo de prova do ensaio de tração seguiram dimensões de acordo com a [27] e os provas de prova do ensaio de impacto izod, seguiram [28]. Os parâmetros de processamento são expostos na tabela 1. A figura 3 demonstra os corpos de prova obtidos para o ensaio de tração. 


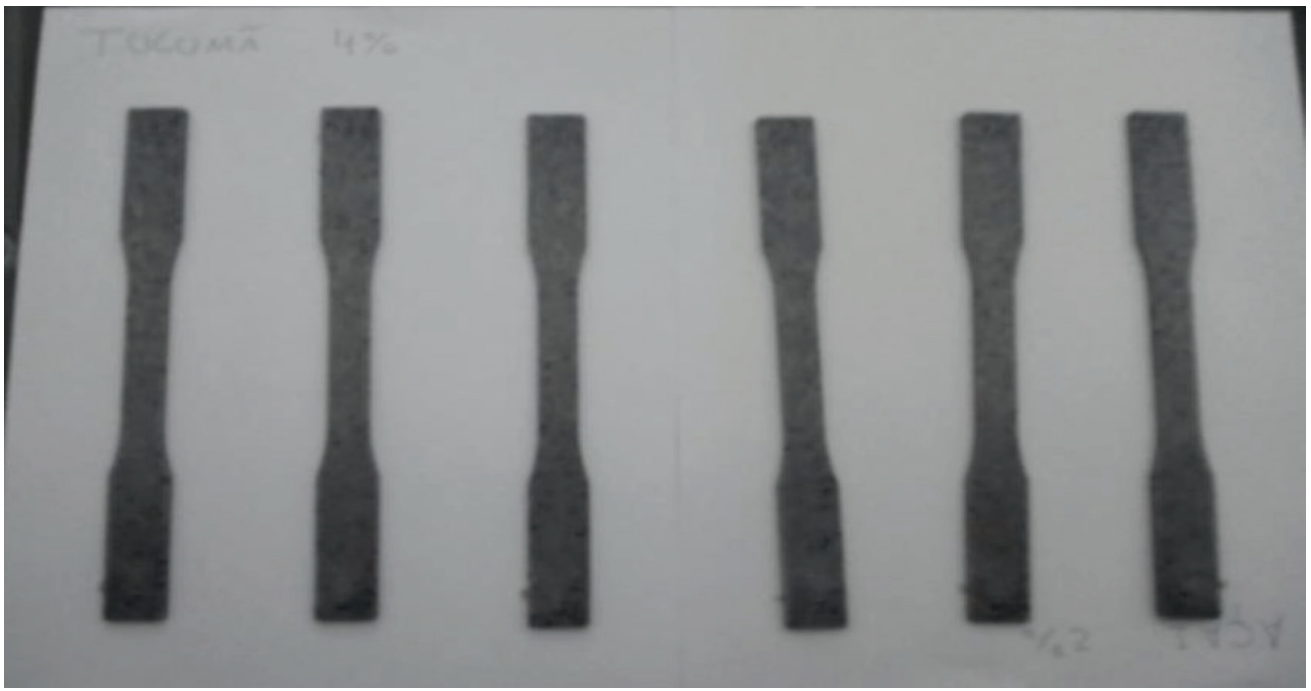

Figura 3: Corpos de provas de tração obtidos via processamento de injeção plástica.

Tabela 1: Parâmetros do equipamento para injeção do PSAI.

\begin{tabular}{cccc}
\hline Temperatura $\left({ }^{\circ} \mathbf{C}\right)$ & Alimentação & Compressão & Dosagem \\
\hline Teórica & 200 & 225 & 230 \\
Real & 204 & 224 & 229 \\
\hline
\end{tabular}

\subsection{Caracterização}

A resistência ao impacto IZOD foi ensaiada conforme ASTM D 256 [28], corpos de prova com entalhe, em um equipamento Tinius Olsen 899 Specimen Notcher. O ensaio de resistência à tração foi realizado em uma máquina universal Equilam EQTB-100 configurado conforme a norma ASTM D638 [27] com velocidade de afastamento de $10 \mathrm{~mm} / \mathrm{min}$.

As análises de TG (análise termogravimétrica) e DSC (calorimetria exploratória diferencial) foram realizadas em um analisador térmico simultâneo TG-DSC da TA Instruments, modelo SDT Q600. As análises foram feitas em cadinho de alumina com cerca de $10 \mathrm{mg}$ de amostra em uma faixa de temperatura de 0 a $600^{\circ} \mathrm{C}$ sob atmosfera de $\mathrm{N}_{2}$ (fluxo de 30 $\mathrm{mL} \cdot \mathrm{min}^{-1}$ ) e razão de aquecimento de $10^{\circ} \mathrm{C} / \mathrm{min}$.

As análises de espectroscopia de infravermelho foram obtidas através de um espectrômetro Thermo Electron (modelo Nicoleti iS10), com 32 varreduras na faixa de 4000 a $400 \mathrm{~cm}^{-1}$ e resolução de $4 \mathrm{~cm}^{-1}$. Cada amostra foi misturada com $\mathrm{KBr}$ na proporção de 1:100 e prensada em disco na pressão $80 \mathrm{KN}$ por $1 \mathrm{~min}$.

\section{RESULTADOS E DISCUSSÃO}

\subsection{Propriedades Mecânicas}

A figura 4 mostra o resultado das analises de tensão-deformação dos seguintes materiais CPS2, CPS4 e PSAI. A resposta de maior resistência à tração foi observada na amostra PSAI, a curva mostrou um comportamento termoplástico característico, como uma região elástica linear e elevada deformação na ruptura.

Observa-se que adição do resíduo de tucumã na composição ocorre consequente diminuição no valor da tensão máxima e uma redução significativa no estiramento. Este fato pode ser associado à fraca adesão entre o resíduo e o polímero. Um aumento na fração volumétrica de resíduo tende a aumentar a região interfacial entre os dois componentes, quanto maior a fração de resíduo, maior a tendência de uma distribuição heterogênea [10]. Por consequente, observar-se um aumento no módulo elástico em CPS2 e CPS4, como é reportado na literatura, o módulo elástico está correlacionada à rigidez do material, a adição de resíduo de tucumã (Astrocaryum aculeatum) torna o material mais rígido. 


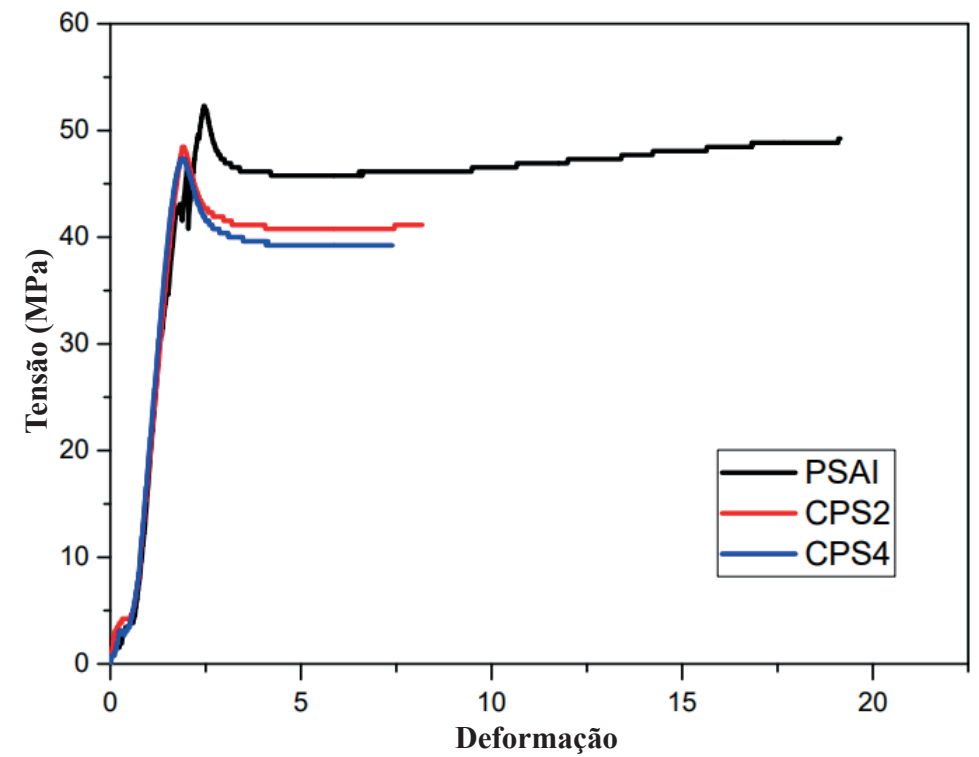

Figura 4: Curvas de Tensão x Deformação média para os corpos de prova PSAI, CPS2 e CPS4.

Os valores médios de resistência máxima em tração, módulo de elasticidade e resistência ao impacto, estão expostas na tabela 2. A adição do resíduo de tucumã provocou a diminuição do alongamento na ruptura, afetando à tração, porém todas as amostras apresentam uma região de escoamento [16,17].

Tabela 2: Propriedades Mecânicas

\begin{tabular}{cccc}
\hline Amostra & $\begin{array}{c}\text { Resistência a Tração } \\
(\mathrm{MPa})\end{array}$ & $\begin{array}{c}\text { Módulo de Elasticidade } \\
(\mathrm{GPa})\end{array}$ & $\begin{array}{c}\text { Resistência ao Impacto } \\
\left({\mathrm{J} . m^{-1}}^{-1}\right.\end{array}$ \\
\hline PSAI & 53,0 & 2,20 & 170,8 \\
CPS2 & 49,6 & 2,02 & 109,7 \\
CPS4 & 48,0 & 2,19 & 118,3 \\
\hline
\end{tabular}

Através de ensaio de impacto, como observado na figura 5, é possível notar que a adição de resíduo de tucumã, modificou levemente o comportamento mecânico dos materiais. Quando se compara com o material puro, PSAI observa-se uma redução de $30 \%$ da resistência ao impacto. Segundo $[16,17]$ a perda de energia absorvida é associada à interface de resíduo-polímero, que pode gerar regiões de fratura.

Porém quando se analisa apenas o CPS2 e CPS4, observa-se um aumento de tenacidade, quando aumenta a fração de resíduo, este processo pode ser associado a um aumento da resposta do resíduo em absorver energia (resposta elástica), como evidenciando [17]. 


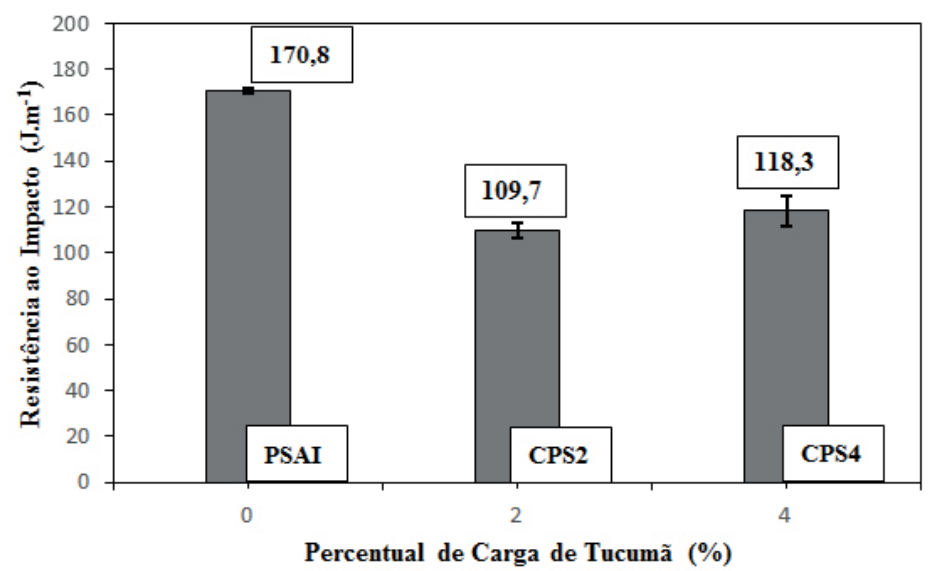

Figura 5: Valores médios do ensaio de impacto.

Cabe salientar que os valores obtidos de propriedades mecânicas estão dentro de uma margem aceita para processo de manufatura, conforme a referência $[18,19,20]$. E de maneira macromolecular, observa-se uma boa adesão entre as partículas de resíduo com o material polimérico, o qual pode ser associado à lignina residual, que atua com agente compatibilizante.

\subsection{Análise Térmica}

As curvas de calorimetria diferencial de varredura (DSC) dos materiais são expostas na Figura 6, destacam próximo a 90 ${ }^{\circ} \mathrm{C}$, para as amostras de PSAI, CPS2 e CPS4 um evento termodinâmico de segunda ordem responsável pelo começo do amolecimento dos segmentos das cadeias, chamado de temperatura de transição vítrea (Tg) [21].

O próximo evento é registrado como um pico exotérmico próximo a $200{ }^{\circ} \mathrm{C}$, sendo este o ponto onde, além a parte amorfa, a parte cristalina do material polimérico é afetada pela quebra de ligações secundárias de Van Der Walls permitindo assim o movimento translacional das cadeias. Este ponto é chamado de temperatura de fusão (Tm), é um evento termodinâmico de primeira ordem [21]. O pico endotérmico na região de $430{ }^{\circ} \mathrm{C}$ é correlacionado a evaporação de produtos voláteis, seguido de dois picos exotérmicos de degradação do polímero. Com a adição de tucumã a essa matriz polimérica é possível observar um deslocamento das curvas de Tm para direita, o que torna o material mais resistente à temperatura (ou a passagem de calor). A curva marrom expõe eventos que ocorrem no reforço (tucumã), os quais são mais bem visualizados nas curvas de TG.

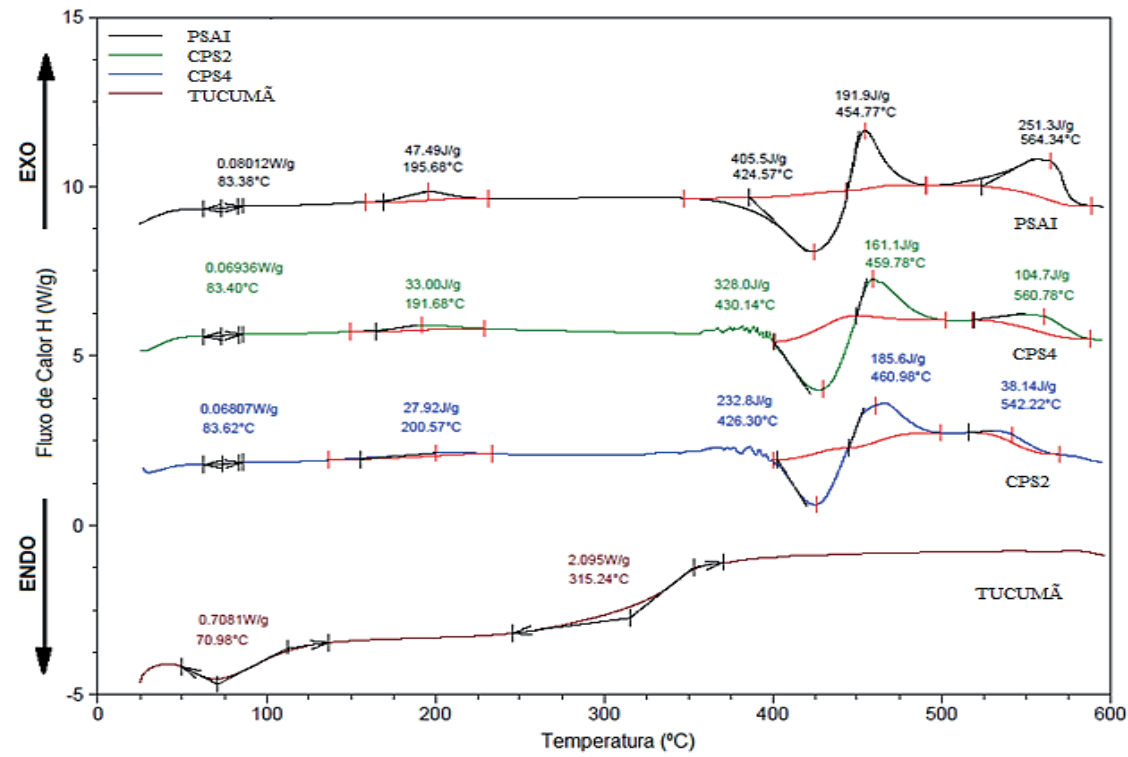

Figura 6: Curvas da Análise Diferencial Exploratória. 
A Figura 7 apresenta as curvas de termogravimétricas do PSAI e dos compósitos de 2 e $4 \%$ em peso de tucumã. Onde é possível observar que esses apresentaram apenas uma variação de massa, correspondente a decomposição vista também nas curvas de DSC, na faixa de 389 a $468^{\circ} \mathrm{C}$. As curvas termogravimétricas do PSAI e dos materiais CPS2 e CPS4, confirmam o deslocamento para a direita observada nos picos da DSC.

Para a curva termogravimétrica do tucumã (Figura 8) é possível observar a perda de água de 25 a $100{ }^{\circ} \mathrm{C}$, correspondente a $10,59 \%$ de massa, seguido de uma estabilidade até $250^{\circ} \mathrm{C}$. O material entra em combustão, perdendo celulose e hemicelulose de $(250$ a 350$){ }^{\circ} \mathrm{C}$, inerente a $23,79 \%$ de massa. E por fim, tem-se a perda de lignina de $(350$ a 550$){ }^{\circ} \mathrm{C}$, correlacionado a $26,75 \%$ de massa. Gerando cinzas de $38,87 \%$ de massa ao final da análise $[22,23,24]$.

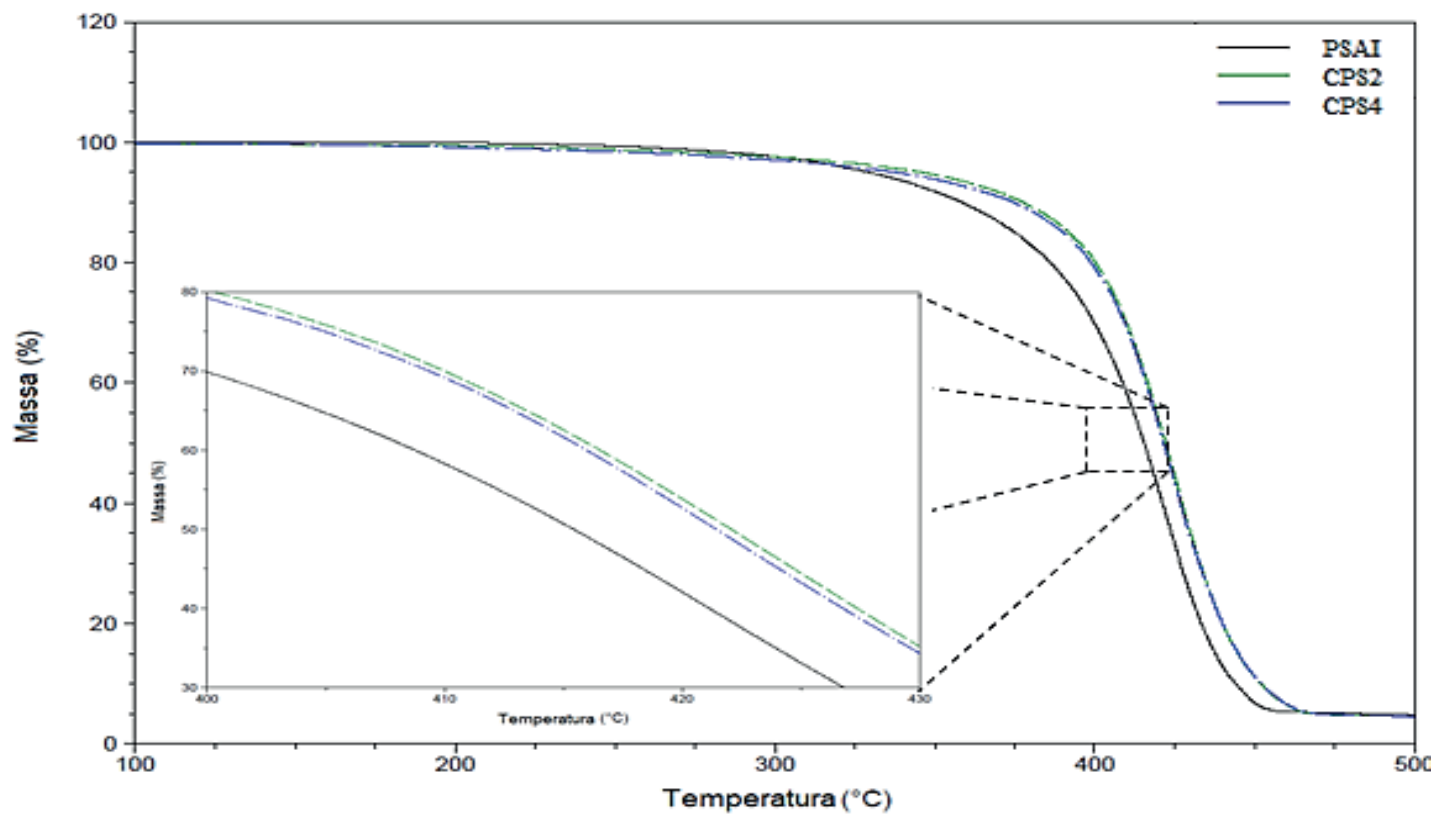

Figura 7: Curvas da Análise Termogravimétrica.

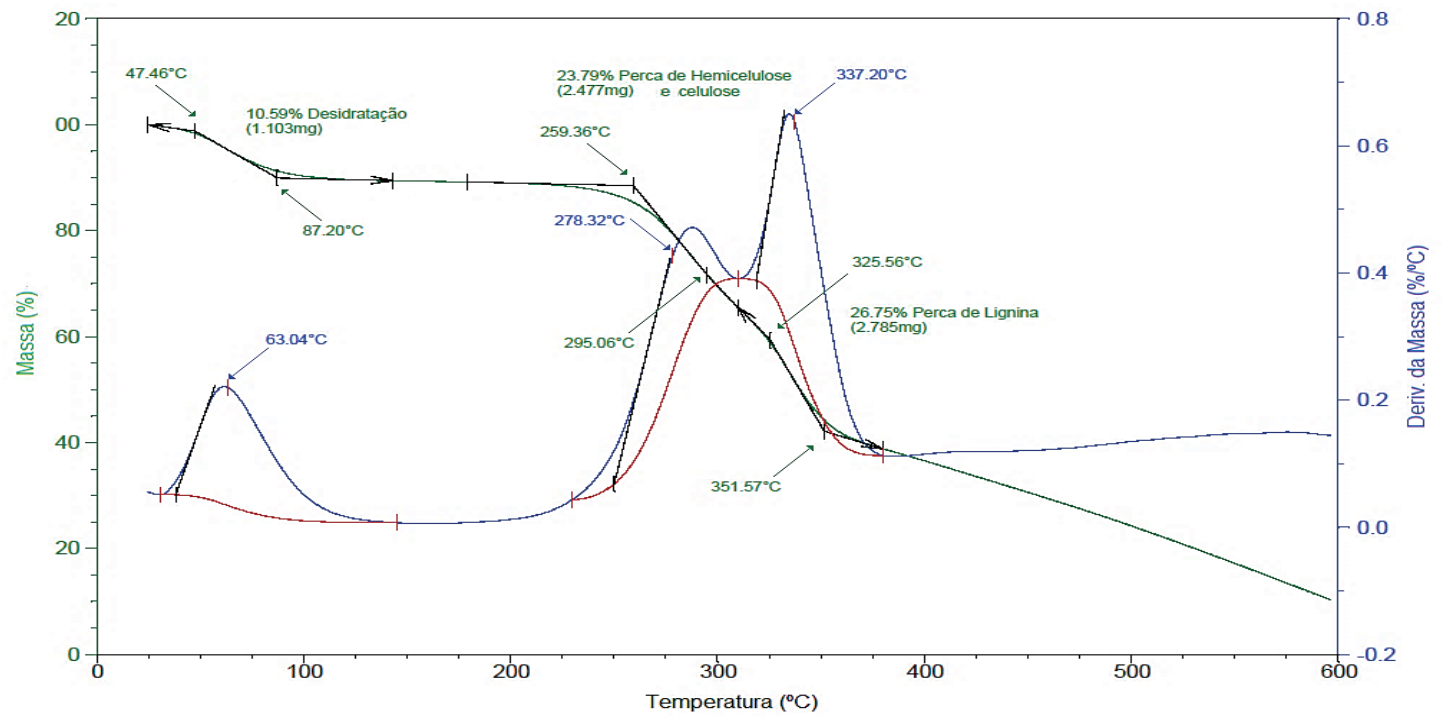

Figura 8: Curvas de TG e DTG do tucumã. 


\subsection{Espectroscopia de Infravermelho}

Na Figura 8 observam-se espectros de infravermelho com transformada de fourier (FTIR) para as amostras PSAI, TUCUMÃ, CPS2 e CPS4. Na referida análise foi possível constar vibrações de grupo moleculares próximo de $3.500 \mathrm{~cm}^{-1}$ referente a estiramentos de $\mathrm{O}-\mathrm{H}$ associado à umidade nas amostras. No polímero puro (PSAI) esse estiramento é mais acentuado, nas amostras de tucumã e nos (CPS2 e CPS4) notasse com menor intensidade.

$\mathrm{Na}$ amostra tucumã observa-se uma banda na região de $2.750 \mathrm{~cm}^{-1} \mathrm{o}$ qual indica ligações $\mathrm{O}-\mathrm{H}$, esse comportamento é observado na FTIR dos compósitos (CPS2 e CPS4). No material puro (PSAI) nota-se estiramento relacionado aos grupos aromáticos (1.600- $1500 \mathrm{~cm}-1)$ bem como vibrações de componentes elastoméricos. Próximo de $1.200 \mathrm{~cm}^{-1}$ observa-se ligação de C-O correspondente a vibrações de grupos fenólicos, está mesma banda vibracional foi observada em CPS2 e CPS4. Em 700- $720 \mathrm{~cm}^{-1}$ observa-se uma alta intensidade com relação à monossubstituição de aromática na amostra PSAI, porém esse mesmo comportamento não pode ser constado nas demais amostras. [25, 26]

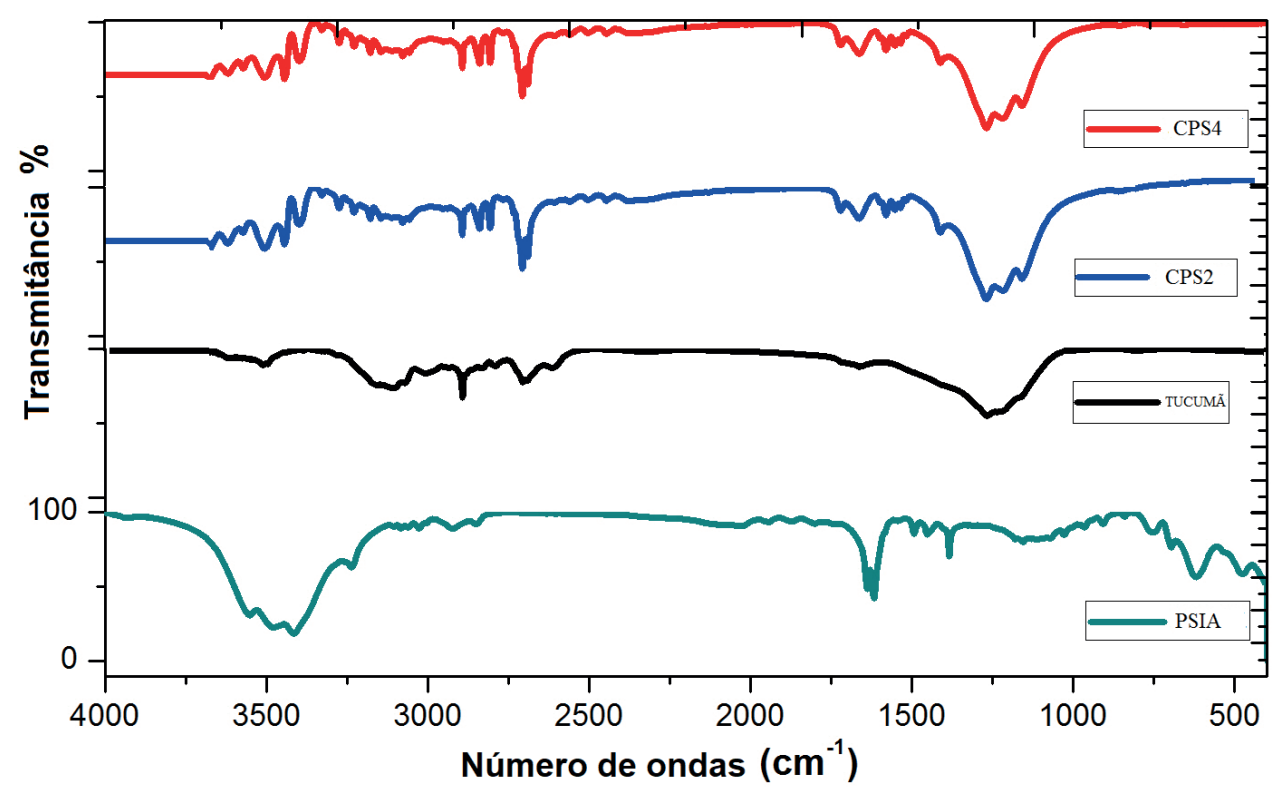

Figura 9: Espectros de FTIR.

A ausência dos picos de $720 \mathrm{a} 700 \mathrm{~cm}^{-1}$ no CPS2 e CP4 indica uma perda das ligações referentes à monossubstituição do anel benzênico. Comparado às curvas de DSC (Figura 6), pode-se afirmar que a perda dessas ligações mais fracas é evidenciada nos picos exotérmicos acima de $500^{\circ} \mathrm{C}$, pois notasse uma redução drástica do fluxo de calor nesse ponto. Assim sendo, a adição de carga de tucumã ao PSAI, tende a diminuir a presença do anel benzênico na estrutura molecular do compósito, o que explicaria a perda de resistência mecânica. Esta perda das ligações de monossubstituição é confirmada pelo decaimento da intensidade dos picos de 1500 a $1600 \mathrm{~cm}^{-1}$, que está relacionado a grupos aromáticos (ou seja, anéis benzênicos). A presença de picos mais acentuados nas faixas de $2750 \mathrm{~cm}^{-1}$ nos CPS2 e CPS4 está correlacionada às muitas ligações O-H de origem hemicelulósica advinda do tucumã. [26]

\section{CONCLUSÕES}

A utilização do endocarpo do tucumã se tornou uma alternativa na obtenção de compósitos plásticos de madeira (WPC). De forma macromolecular foi possível observar uma boa adesão entre as partículas, à lignina residual pode ter atuado como agente compatibilizante possibilitando a aumento de interação entre resíduo e poliestireno de alto impacto. Essas interações proporcionaram a alteração nas propriedades térmicas e mecânicas. As análises mecânicas indicam que os materiais com adição do resíduo tiveram um decréscimo de absorção de energia, porém dentro de uma faixa engenharia pode ser utilizado como futuro substituinte plástico. Do ponto de vista térmico, é observado que a presença do resíduo produz um aumento nos valores de transições termodinâmicas. Por meio da análise de infravermelho, observamos grupos funcionais dos materiais, bem como seu ambiente químico. Fato este evidenciado pela perda parcial de ligações monossubstituidas dos anéis aromáticos em CP2 e CP4. Portanto, conclui-se que a utilização do resíduo do endocarpo do tucumã com o poliestireno de alto impacto, se torna viável, na substituição de materiais plásticos, dependendo da aplicação e condições estruturais de projeto. 


\section{AGRADECIMENTOS}

Ao Laboratório de Síntese e Caracterização de Nanomateriais (LSCN/IFAM), na pessoa do técnico Mitsuo Lopes. Ao Laboratório de Ensaios Mecânicos do IFAM, ao Laboratório de Materiais da Amazônia e Compósitos (LAMAC/UFAM). Ao Laboratório de Espectroscopia de Infravermelho da Central Analítica da UFAM. Aos feirantes da feira do produtor da cidade de Manaus pela doação dos materiais da pesquisa. E por fim, a Videolar - Innova S/A- Unidade IV, na pessoa da gerente industrial Adriana.

\section{BIBLIOGRAFIA}

[1] JESUS, L.C.C.; LUZ, S.M.; LEÃO, R.M., et al., "Comportamento térmico de compósitos de poliestireno reciclado reforçado com celulose de bagaço de cana.” Revista Matéria, v.24, n. 3, 2019.

[2] IDICULA, M., MALHOTRA, S.K., KURUVILlA, J., et al., "Dynamic mechanical analysis of randomly oriented intimately mixed short banana/sisal hybrid fibre reinforced polyester composites." Composites Science and Technology, v.65, n. 7, pp. 1077-1087, 2005.

[3] WEARN, Y.N., MONTAGNA, L.S., PASSADOR, F. R. "Compósitos de fibra de coco/LDPE: efeito do tratamento superficial das fibras de coco em compósitos verdes”. Matéria (Rio de Janeiro), 25(1), e-12548. Epub April 06, 2020.

[4] MARINELLI, A. L., et al., "Desenvolvimento de compósitos poliméricos com fibras vegetais naturais da biodiversidade: uma contribuição para a sustentabilidade amazônica.” Polímeros, v.18, n.2, 2008.

[5] SHERMAN, V. R., QUAN, H., YANG,W., el al. "Estudo comparativo da defesa dos peixes: as escalas de Arapaima gigas, Latimeria chalumnae e espátula de Atractosteus.” J Mech Behav Biomed Mater. 2017.

[6] KIELING, A. C., SANTANA, G. P. "Compósito fabricado do endocarpo do tucumã (Astrocaryum Aculeatum) com Polímero Termoplástico”. Scientia Amazonia, ISSN: 2238.1910, v. 6, n.3, pp. 24-30, 2017.

[7] XIE, Q., LI, F., LI, J., el al. “A new biodegradable sisal fiber-starch packing composite with nest structure”. Carbohydrate polymers, v. 189, pp. 56-64. 2018.

[8] SILVA,R.S.,SANTOS,C.L.,MAR,J.M., et al. “ Physicochemical properties of tucumã (Astrocaryum aculeatum) powders with different carbohydrate biopolymers”, LWT, v. 94, 2018.

[9] SANTOS, R.C.V., SAGRILLO, M. R., RIBEIRO, E.E., et al.; "The Tucumã of Amazonas- Astrocaryum aculeatum”. Exotic Fruits. Pages, pp. 419-425, 2018.

[10] TORRE, G. B., DOGNANI, G., CABRERA, F.C., et al. "Sustainable blends of LDPE/NR and sugarcane bagasse ashes with PE-g-MA thermomechanical relationships". Matéria (Rio de Janeiro), 24(3), e12411. Epub September 16, 2019.

[11] LUNA, C. B. B., GOMES, F.B.C., SIQUEIRA, D.D., et al. "Influência do envelhecimento termo-oxidativo nas propriedades mecânicas e de amarelamento de blendas de poliestireno com borracha reciclada de estireno-butadieno (SBR)". Matéria (Rio de Janeiro), 24(3), 12414. Epub September 16, 2019.

[12] HØGSAA, B., PEDERSEN, T.H., MOUSAVI, M. et al. "Multiscale Characterization of a Wood-Based Biocrude as a Green Compatibilizing Agent for High-Impact Polystyrene/Halloysite Nanotube Nanocomposites". ACS Omega, v. 4, pp. 19934-19943, 2019.

[13] DING, L., JIA, Z., SUN, H., et al. "Estimation of Mechanical Performance, Thermal Stability and Flame Retardancy of High-Impact Polystyrene/Surface-Modified APP/Carboxylic-Functionalized MWCNTs Nanocomposites”. Polymers, v. 11, pp. 615. 2019.

[14] MOTTA, L. A.C., GONÇALVES, L. K.S., SILVA, M. R., et al. "Painéis sanduíches de poliéster reforçado com fibras de rami para aplicação na Construção Civil”. Matéria (Rio de Janeiro), v. 21, n. 3, pp. 796-806. 2016.

[15] PACHLA, E., SILVA, D., JUCÁ, P., et al. "Desempenho térmico-acústico-mecânico de um compósito de matriz cimentícia leve reforçado com casca de arroz”. Matéria (Rio de Janeiro), 24(2), e12354. Epub June 10, 2019

[16] CLAUS, J., SANTOS, R.A.M., GORBATIKH, L., et al."Effect of matrix and fibre type on the impact resistance of woven composites”. Composites Part B: Engineering,V. 183, 2020.

[17] VARUN, K.T., PRASAD, V. M. M., SANTHOSH, D., et al."Evaluation of mechanical and interfacial properties of carbon fiber reinforced polymer (CFRP) composite materials”.Materials Today: Proceedings, Volume 21, Part 1, 2020. 
[18] CAPELIN, L.J., MORAES, K. K., ZAMPIERI, J. P., et al.“Avaliação dos efeitos da fibra de coco e da microcelulose cristalina nas propriedades de argamassas cimentícias”. Matéria (Rio de Janeiro), v. 25, n. 1, e-12551. Epub April 06, 2020

[19] RIBEIRO, V. F., DOMINGUES, JR., NEI, S.; et al. "Estudo da recuperação das propriedades de poliestireno de alto impacto (HIPS) através da incorporação de borracha termoplástica tipo estireno-butadieno-estireno (SBS) ".Polímeros, São Carlos , v. 22, n. 2, p. 186-192, 2012.

[20] GRASSI, V.G., FORTE, M.M.C., DAL, P., MARCUS, F., “Aspectos Morfológicos e Relação Estrutura-Propriedades de Poliestireno de Alto Impacto”. Polímeros, São Carlos, v. 11, n.3, pp. 158-168, Sept. 2001.

[21] LUNA, C. B. B., SILVA, D. F., ARAÚJO, E. M., et al. "Estudo do Comportamento Mecânico, Termomecânico e Morfológico de Misturas de Poliestireno/Composto de Borracha Reciclada (SBR)". Matéria (Rio de Janeiro), v. 20, n. 2 , pp. 322-334, 2015.

[22] MEIRELES, C.D.S., FILHO, G.R., ASSUNÇÃO, R.M.N., et al. "Blend compatibility of waste materials-Cellulose acetate (from sugarcane bagasse) with polystyrene (from plastic cups): Diffusion of water, FTIR, DSC, TGA, and SEM study”. J. Appl. Polym. Sci., v. 104, pp. 909-914. 2007.

[23] VILAPLANA, F., RIBES-GREUS, A., KARLSSON,S., "Degradation of recycled high-impact polystyrene. Simulation by reprocessing and thermo-oxidation”.Polymer Degradation and Stability, v. 91, Issue 9, 2006.

[24] ZHANG, X., HAO, X., HAO, J., et al.“Thermal and mechanical properties of wood-plastic composites filled with multiwalled carbon nanotubes”. Journal of Applied Polymer Science, 135(22), 46308. 2018.

[25] MARTÍNEZ-BARRERA, G., LÓPEZ, H., CASTAÑO, V.M., et al."Studies on the rubber phase stability in gamma irradiated polystyrene-SBR blends by using FT-IR and Raman spectroscopy". Radiation Physics and Chemistry, Volume 69, Issue 2, 2004.

[26] LEONARDI, B; ARAUZ, L.J; BARUQUE, R, J. “Chemical characterization of amazonian non-polar vegetal extracts (Buriti, Tucumã, Brazil Nut, Cupuaçu, And Cocoa) by infrared spectroscopy (ftir) and gas chromatography (gc-fid) ”. Infarma - Ciências Farmacêuticas, [S.1.], v. 31, n. 3, p. 163-176, oct. 2019.

[27] AMERICAN SOCIETY FOR TESTIND AND MATERIALS. ASTM - D638: Standard test method for tensile properties of plastics, 2014.

[28] AMERICAN SOCIETY FOR TESTIND AND MATERIALS. ASTM - D256: Standard Test Methods for determining the Izod Pendulum Impact Resistance of Plastics, 2018.

\section{ORCID}

Andrey Marcos Pinho da Silva

Daniel Esquerdo Margalho

Darbens Silvio Correa Junior https://orcid.org/0000-0003-1769-3862

https://orcid.org/0000-0002-5918-6810

https://orcid.org/0000-0001-9540-1779 